\title{
Structure, Stability and ELM Dynamics of the H-Mode Pedestal in DIII-D
}

M. E. Fenstermacher, A. W. Leonard, T. H. Osborne, P. B. Snyder, D. M. Thomas, J. A. Boedo, T. A. Casper, R. J. Colchin, R. J. Groebner, M. Groth, M. A. H. Kempenaars, A. Loarte, G. Saibene, M. A. VanZeeland, L. Zeng, X. Q. Xu

October 14, 2004

20th IAEA Fusion Energy Conference Vilamoura, Portugal

November 1, 2004 through November 6, 2004 
This document was prepared as an account of work sponsored by an agency of the United States Government. Neither the United States Government nor the University of California nor any of their employees, makes any warranty, express or implied, or assumes any legal liability or responsibility for the accuracy, completeness, or usefulness of any information, apparatus, product, or process disclosed, or represents that its use would not infringe privately owned rights. Reference herein to any specific commercial product, process, or service by trade name, trademark, manufacturer, or otherwise, does not necessarily constitute or imply its endorsement, recommendation, or favoring by the United States Government or the University of California. The views and opinions of authors expressed herein do not necessarily state or reflect those of the United States Government or the University of California, and shall not be used for advertising or product endorsement purposes. 


\title{
Structure, Stability and ELM Dynamics of the H-Mode Pedestal in DIII-D
}

\author{
M.E. Fenstermacher, ${ }^{1}$ A.W. Leonard, ${ }^{2}$ T.H. Osborne,${ }^{2}$ P.B. Snyder, ${ }^{2}$ D.M. Thomas, ${ }^{2}$ \\ J.A. Boedo, ${ }^{3}$ T.A. Casper, ${ }^{1}$ R.J. Colchin, ${ }^{4}$ R.J. Groebner, ${ }^{2}$ M. Groth, ${ }^{1}$ M.A.H. Kempenaars, ${ }^{5}$ \\ A. Loarte, ${ }^{6}$ G. Saibene, ${ }^{6}$ M.A. VanZeeland,${ }^{2}$ L. Zeng, ${ }^{7}$ X.Q. Xu, ${ }^{1}$ and the DIII-D Team \\ ${ }^{1}$ Lawrence Livermore National Laboratory, P.O. Box 808, Livermore, California, USA \\ ${ }^{2}$ General Atomics, P.O. Box 85608, San Diego, California 92186-5608, USA \\ ${ }^{3}$ University of California, San Diego, California, USA \\ ${ }^{4}$ Oak Ridge National Laboratory, Oak Ridge, Tennessee 37831, USA \\ ${ }^{5}$ FOM-Rijnhuizen, Assoc. Euratom-FOM, TEC, Nieuwegein, Netherlands \\ ${ }^{6}$ EFDA-CSU, Max-Planck-Institut for Plasmaphysik, D-85748 Garching, Germany \\ ${ }^{7}$ University of California Los Angeles, Los Angeles, California, USA \\ e-mail contact of main author: max.fenstermacher@gat.com
}

\begin{abstract}
Experiments are described that have increased understanding of the transport and stability physics that set the H-mode edge pedestal width and height, determine the onset of Type-I edge localized modes (ELMs), and produce the nonlinear dynamics of the ELM perturbation in the pedestal and scrape-off layer (SOL). Predictive models now exist for the $n_{\mathrm{e}}$ pedestal profile and the $\mathrm{p}_{\mathrm{e}}$ height at the onset of Type-I ELMs, and progress has been made toward predictive models of the $T_{\mathrm{e}}$ pedestal width and nonlinear ELM evolution. Similarity experiments between DIII-D and JET suggested that neutral penetration physics dominates in the relationship between the width and height of the $n_{\mathrm{e}}$ pedestal while plasma physics dominates in setting the $T_{\mathrm{e}}$ pedestal width. Measured pedestal conditions including edge current at ELM onset agree with intermediate-n peeling-ballooning (P-B) stability predictions. Midplane ELM dynamics data show the predicted (P-B) structure at ELM onset, large rapid variations of the SOL parameters, and fast radial propagation in later phases, similar to features in nonlinear ELM simulations.
\end{abstract}

\section{Introduction}

This paper describes experiments that were focused on optimizing pedestal parameter measurements to determine the transport and stability physics that set the H-mode edge pedestal width and height, the onset conditions for Type-I edge localized mode (ELM) instabilities, and the nonlinear dynamics of the ELM perturbation observed in the pedestal and midplane scrape-off layer (SOL). These are critical issues for future burning plasma devices such as ITER because for stiff profiles the height of the pedestal determines the overall confinement [1], and the size of the ELMs determines divertor target lifetimes [2]. The experiments were carried out primarily onDIII-D with additional results coming from dimensionally similar plasmas in DIII-D and JET.

Results are in agreement with predictive models for the density pedestal width and the pressure gradient at the onset of Type-I ELMs, and show that significant progress has been made toward generating predictive models of the temperature pedestal width (transport barrier) and nonlinear ELM evolution. The measurements show that neutral penetration physics is playing a significant role in setting the density pedestal parameters. The pressure pedestal gradient is limited by the stability of coupled peeling-ballooning (P-B) instabilities at the edge. In similarity experiments with fixed pedestal beta, $\beta$, collisionality, $v^{*}$, normalized gyroradius, $\rho^{*}$ and safety factor, $\mathrm{q}$, the transport barrier width, $\Delta T$, scaled with minor radius, a. When $\rho^{*}$ was varied at fixed $\left(\beta, \nu^{*}, \mathrm{q}\right), \Delta T / a$ was nearly independent of $\rho^{*}$, and ELM size decreased as $\rho^{*}$ decreased in agreement with changes in the radial mode width of the most unstable P-B mode. New edge current measurements confirmed the edge bootstrap current models used in the edge stability calculations. Finally, the initial data from non-linear ELM simulations indicated that ELMs have a complicated spatial and temporal structure in the pedestal and SOL. Some initial scaling of these results to future devices is possible, as described below.

The paper is organized as follows. The experimental techniques and some of the important diagnostic measurements are described in Sec. 2. Experimental results are 
described in Sec. 3 including those from the pedestal similarity experiments, the edge stability characterization, and the nonlinear ELM dynamics. A summary and conclusions are given in Sec. 4. 


\section{Experimental Techniques and Diagnostics}

The pedestal transport and stability mechanisms were investigated both with new diagnostics in DIII-D and in similarity experiments with matched plasma shape and dimensionless pedestal parameters between DIII-D and JET. The similarity experiments focused on determining the physics mechanisms that set the pedestal widths. These were done in matched lower single-null (LSN) discharges with optimized shapes for pedestal profile diagnostics on JET (DOC-L shape with elongation $\kappa=1.72$ and average triangularity, $\delta_{\text {avg }}=0.27$ and DOC-U shape with $\kappa=1.68$ and $\delta_{\text {avg }}=0.40$ ). For JET, typical discharge parameters were plasma current $I_{\mathrm{p}}=1.2-2.5 \mathrm{MA}$, major radius $\mathrm{R}=2.95 \mathrm{~m}$, minor radius a $=0.93 \mathrm{~m}$, and heating power in the range $P_{\text {inj }}=4.9-17.0 \mathrm{MW}$. For the pedestal similarity experiments, the dimensionless parameters $\beta \sim n T / B_{\mathrm{T}}$ effective collisionality, $v^{*} \sim n q R A^{3 / 2} / T^{2}$, effective Larmor radius, $\rho^{*} \sim T^{1 / 2} / a B_{\mathrm{T}}$ and safety factor, $q \sim a^{2} B_{\mathrm{T}} / R I_{\mathrm{p}}$ were matched at the top of the pedestal, although they could not be matched across the entire transport barrier profile. Here $B_{\mathrm{T}}$ is the toroidal field, $q$ is the safety factor at $95 \%$ flux, $n$ and $T$ are the density and temperature respectively, and $A$ is the aspect ratio, $R / a$. In discharges with matched shape, maintaining fixed $\beta, \rho^{*}, \nu^{*}$, and $q$ at the top of the pedestal requires that density, temperature, toroidal field, and plasma current scale as $n^{\text {ped }} \sim a^{-2}, T$ ped $\sim A^{5 / 4} a^{-1 / 2}, B_{\mathrm{T}} \sim A^{5 / 8} a^{-5 / 4}$, and $I_{\mathrm{p}} \sim$ $A^{-3 / 8} a^{-1 / 4}$ respectively. Studies of $\rho^{*}$ dependence were done by varying $B_{\mathrm{T}}$. In this case with fixed $q$, maintaining fixed $\beta$ and $*$ at the top of the pedestal requires that $n^{\text {ped }} \sim$ $A^{-5 / 6} a^{-1 / 3} B_{\mathrm{T}^{-}}^{{ }^{-}}, T^{\text {ped }} \sim A^{5 / 6} a^{1 / 3} B_{\mathrm{T}^{-}}^{-}$and $I_{\mathrm{p}} \sim A^{-1} a B_{\mathrm{T}}$. Toroidal field was varied in JET from $B_{\mathrm{T}}=1.2$ to $2.7 \mathrm{~T}$. Parameters in the DIII-D similarity discharges were $I_{\mathrm{p}}=1.18-1.38 \mathrm{MA}$, $R=1.7 \mathrm{~m}, a=0.6 \mathrm{~m}, P_{\mathrm{inj}} \sim 1.18-9.5 \mathrm{MW}$ and $B_{\mathrm{T}}=1.0$ to $2.1 \mathrm{~T}$. Pedestal profiles were measured in JET with an edge LIDAR system $\left(n_{\mathrm{e}}\right.$ and $\left.T_{\mathrm{e}}\right)$ and with ECE emission $\left(T_{\mathrm{e}}\right)$. On DIII-D, profiles of $n_{\mathrm{e}}$ and $T_{\mathrm{e}}$ were measured with Thomson scattering and the profile of ion temperature $T_{\mathrm{i}}$ was obtained from charge-exchange recombination (CER) spectroscopy.

Pedestal stability physics studies on DIII-D combined detailed pedestal plasma profile measurements with pedestal current density measurements using a unique new diagnostic [3] to predict the onset of ELMs from a linear peeling-ballooning theory with all relevant parameters measured. In these studies the plasma shape was optimized for pedestal and near SOL profile measurements with the DIII-D Thomson scattering and CER systems. Small radial excursions of the separatrix were used to further refine the profile measurements. In addition, for the first time the current density in the pedestal region was directly measured [3] simultaneously with the profiles using polarimetry of an injected lithium beam. Combining magnetics measurements with the measured $n_{\mathrm{e}}, T_{\mathrm{e}}, T_{\mathrm{i}}$, and edge current density provided all the necessary parameters to generate accurate equilibrium reconstructions and to check theories of bootstrap current generation at the edge and peeling-ballooning stability predictions of ELM onset.

Pedestal dynamics during ELMs were measured on DIII-D with simultaneous fast diagnostics near the outer midplane. These included a tangentially viewing radial array of fast $\mathrm{D}_{\alpha}$ detectors at up to $100 \mathrm{kHz}$ [4], a fast reciprocating probe with data acquisition rates of $200 \mathrm{kHz}$ for $n_{\mathrm{e}}$ and $T_{\mathrm{e}}$, and $1 \mathrm{MHz}$ for $I_{\text {sat }}$ [5], profile reflectometry measurements up to $n_{\mathrm{e}}=$ $6 \times 10^{19} \mathrm{~m}^{-3}$ at $40 \mathrm{kHz}$ rate [6], beam emission spectroscopy of radially and poloidally propagating density fluctuations with $1 \mathrm{MHz}$ acquisition rate [7], and a very fast interferometer chord viewing radially in from the outer midplane with a $5 \mathrm{MHz}$ sampling rate $[8]$.

\section{Experimental Results}

\subsection{Pedestal Structure}

Data from similarity experiments between DIII-D and JET suggested that neutral penetration physics dominates in setting the relationship between the width, $\Delta_{\mathrm{n}}$, and height, $n_{\mathrm{e}}^{\mathrm{ycu}}$, of the density pedestal. Density profiles from low-density discharges in the two devices are overlaid in Fig. 1(a) using the scaling for fixed $\beta, \rho^{*}, \nu^{*}$, and $q$. Although the top of the 
$n_{\mathrm{e}}$ pedestal in JET could not be determined precisely, the top of the $n_{\mathrm{e}}$ pedestal in DIII-D was clearly further outboard than in JET. Simulation of these profiles using a neutral penetration model $[9,10]$ reproduced the shape of the profiles including this difference in the radial location of the top of the pedestal [Fig. 1(a)]. The neutral penetration model balances particle diffusion with neutral ionization in the pedestal and SOL and takes into account Franck-Condon neutrals and the effect of poloidal variation in the neutral source due to differences in flux expansion around the SOL. The model predicts that the width of the density pedestal should scale as the inverse of the density at the top of the pedestal, $\Delta_{\mathrm{ne}} \sim 1 / n_{\mathrm{e}}^{\mathrm{ycu}}$. This was observed in higher density similarity plasmas [Fig. 1(c)] in both DIII-D and JET. For both of the density cases, the top of the temperature pedestal [Figs. 1(b,d)] was inboard of the density pedestal in DIII-D. The $n_{\mathrm{e}}$ and $T_{\mathrm{e}}$ profiles were nearly aligned in JET for the low-density conditions [Figs. 1(a,b)] but the top of the $n_{\mathrm{e}}$ pedestal was outboard of the $T_{\mathrm{e}}$ pedestal at high density [Figs. $1(\mathrm{c}, \mathrm{d})]$. These variations in the radial location between the $n_{\mathrm{e}}$ and $T_{\mathrm{e}}$ barriers suggest that physics other than neutral penetration dominates in setting the $T_{\mathrm{e}}$ barrier.

Plasma physics that scales with dimensionless parameters appears to dominate in setting the temperature pedestal width (transport barrier), $\Delta_{\mathrm{T}}$. Some theories suggest that neutral penetration also sets the temperature pedestal width [11]. However, in these pedestal similarity experiments, $\Delta_{\mathrm{T}}$ normalized to the minor radius, $a$, was the same in both machines [Fig. 1(b)], suggesting that plasma physics, not neutral penetration controls the transport barrier width. A similar observation was made in DIII-D/C-Mod similarity experiments [12]. Also consistent with this interpretation was that $\Delta_{\mathrm{T}} \sim a$ was independent of density [Figs. 1 $\left.(\mathrm{b}, \mathrm{d})\right]$.

No obvious variation of $\Delta_{\mathrm{T}} / a$ with $\rho *$ was seen for fixed $\left(\beta, v^{*}, q\right)$ at the top of the pedestal during scans of $B_{\mathrm{T}}$ in DIII-D and JET (Fig. 2). A factor of 2 variation of $\rho *$ was obtained in DIII$\mathrm{D}$ by varying $B_{\mathrm{T}}$ from 1.0 to $2.1 \mathrm{~T}$ and a somewhat smaller variation was obtained in JET. Figure 2 shows no clear dependence on $\rho *$ in the normalized pedestal width, time-averaged over the ELM cycle.

\subsection{Pedestal Stability}

Measured ELM onset conditions compared favorably with ELITE intermediate- $n$ peelingballooning stability constraints calculated in self-consistent equilibria using the measured pedestal plasma profiles and a model for the edge current density, $j$ edge, that was constrained

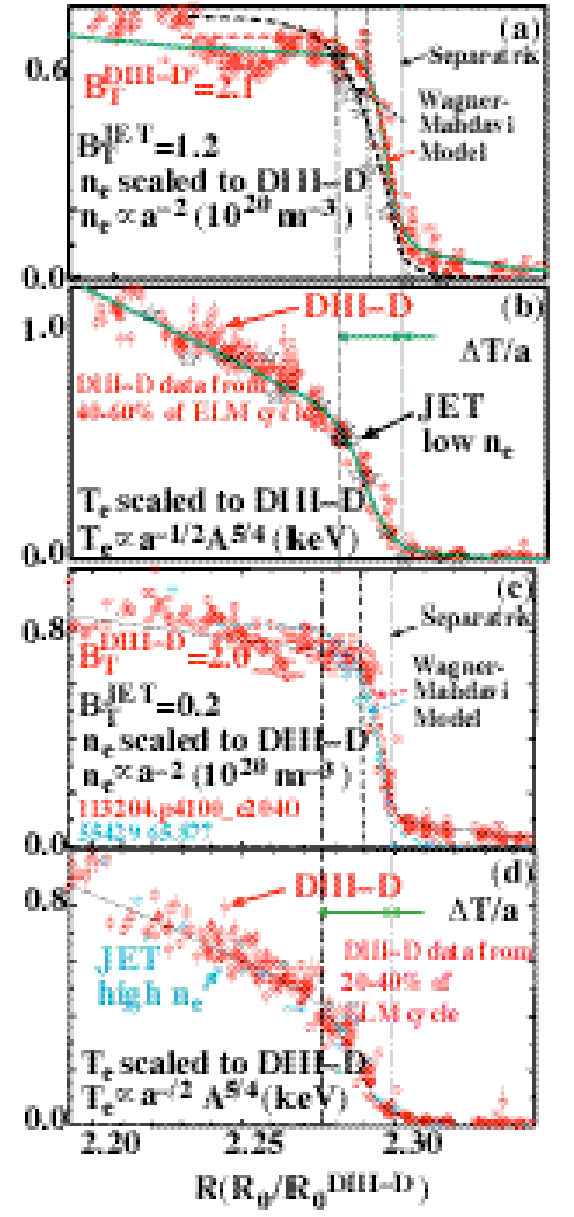

Fig. 1. Comparison of density $(\mathrm{a}, \mathrm{c})$ and temperature $(b, d)$ pedestal profiles in DIII-D and JET for low $(a, b)$ and high (c,d) H-mode operation in the similarity experiments. Pedestal density profiles well predicted by neutral penetration model $(\mathrm{a}, \mathrm{c})$ in both DIII-D and JET. Temperature profile width scales with minor radius $(b, d)$.

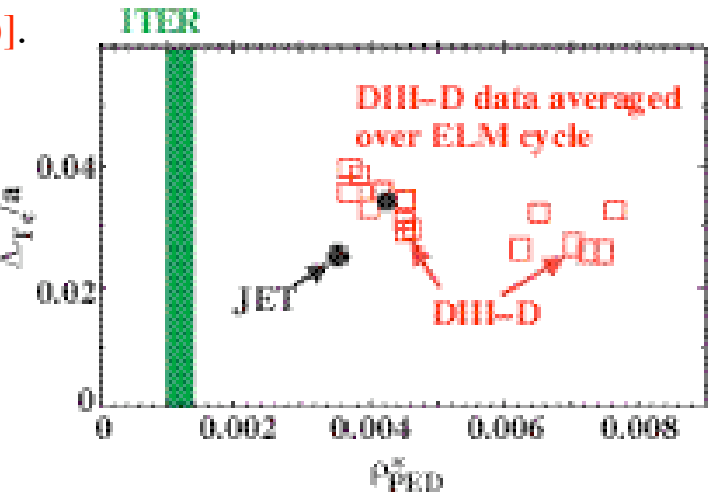

Fig. 2. Temperature pedestal (transport barrier) width as a function of $\rho^{*}$ at the top of the pedestal from DIII-D/JET similarity experiments. Data does not show a strong dependence with $\rho^{*}$. . 
by new $j$ edge measurements (Fig. 3). First direct measurements $[3,13]$ of the poloidal field in the pedestal [Fig. 3(a)], were made at the outer midplane just before ELM onset with a new Libeam polarimetry diagnostic [14]. The inferred jedge [Fig. 3(b)] was consistent with calculations of edge Pfirsch-Schluter and bootstrap currents, using the measured pedestal plasma profiles and the NCLASS bootstrap model [15]. Free boundary equilibria that were constrained by the measured $j$ edge, were generated by the equilibrium solver in the CORSICA code [16]. The inverse solver in CORSICA provided an equilibrium solution in $(\rho, \theta)$ (i.e. poloidal flux, poloidal angle) with high midplane radial and X-point poloidal resolutions using an optimized, non-uniform grid. Linear stability calculations of ELM onset conditions were done on this equilibrium with the ELITE code $[17,18]$. In contrast to ELITE calculations for conditions between ELMs that show stability, for these plasma conditions just before ELM onset, ELITE showed instability for the high $n=30$ 35 modes, stability for low $\mathrm{n} \leq 15$, and marginal stability for intermediate $n$ modes, $16 \leq n \leq 29$. The mode structure for the most unstable mode in this case, $n=25$ is shown in Fig. 4 .

The dependence of the normalized ELM energy loss $\left(\Delta W_{\text {ELM }} / W_{\text {ped }}\right)$, in the DIII-D $\rho^{*}$ scan from the similarity experiments, was consistent with predicted changes in the peeling-ballooning mode width at the edge, but neutral penetration physics also played a role. As $\rho^{*}$ decreased
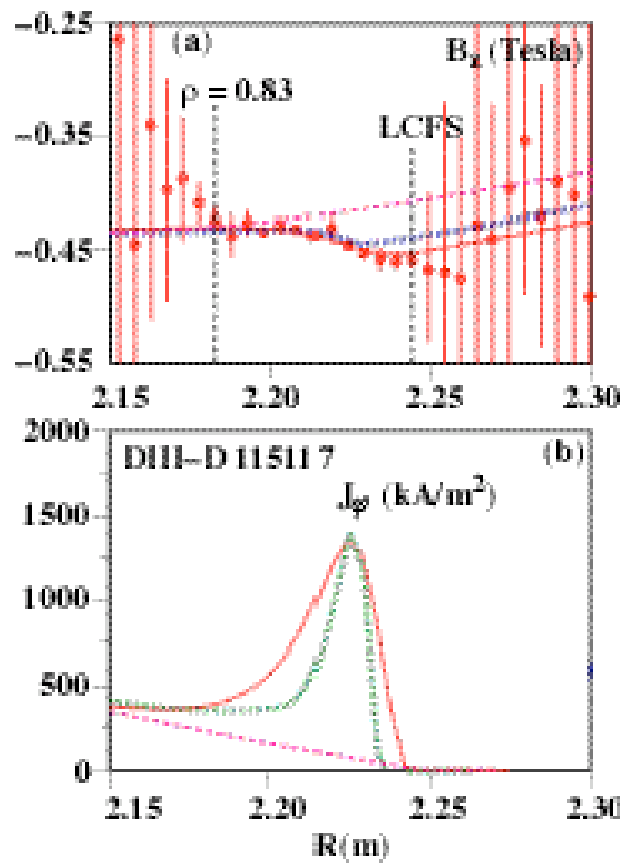

Fig. 3. Li-beam polarimetry measurements of the pedestal poloidal field profile (a), and the inferred edge current density profile (b), for plasma conditions just before ELM onset. The measured profiles (solid) are compared with calculated values using an equilibrium reconstruction code constrained by the NCLASS bootstrap current model (dashed), and measured L-mode phase profiles (dotted). A significant increase in the edge current is seen between L-mode and the profile in H-mode just before an ELM. The measured current density peak of $1.35 \mathrm{MA} / \mathrm{m}^{2}$ agrees well with the calculated value.

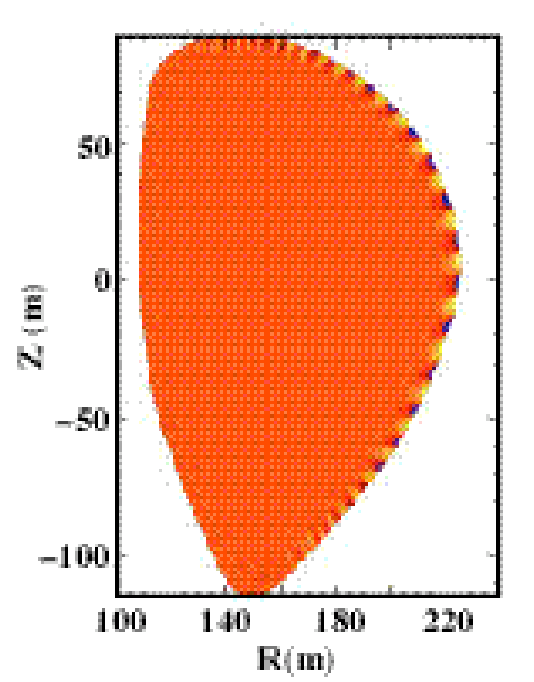

Fig. 4. The ELITE prediction of the poloidal mode structure for the most unstable mode in a kinetic equilibrium reconstructed using the edge current from Fig. 3 as a constant.

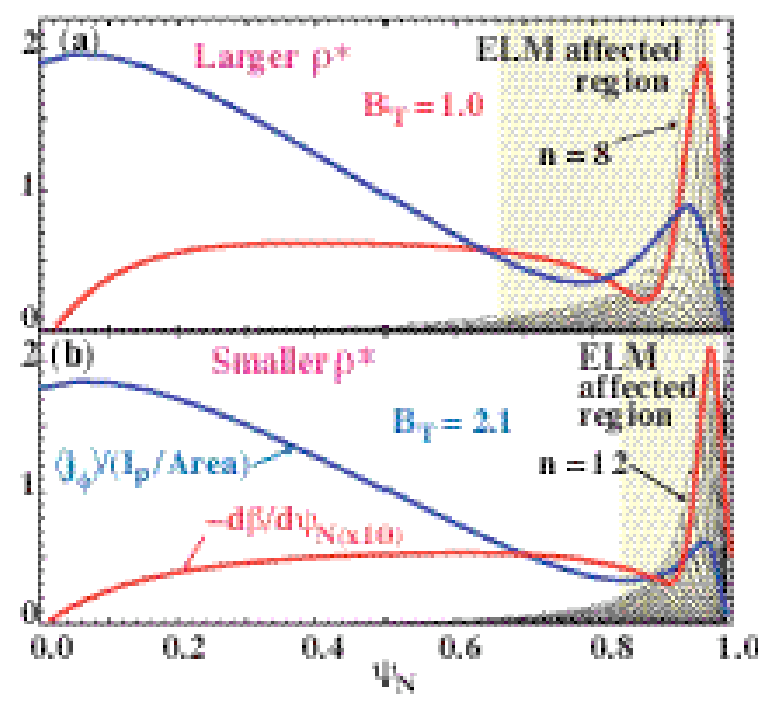

Fig. 5. Measured pressure profile, calculated current profile and most unstable peeling-ballooning mode eigenfunctions for large (a) and small (b) $\rho^{*}$ from the DIII-D/JET similarity experiments. The ELM affected area is reduced for smaller $\rho^{*}$. 
(Fig. 5) the steep gradient region in the measured pressure profile narrowed. The measured plasma profiles before and after ELMs also showed a narrower ELM affected region and reduced ELM energy loss at low $\rho^{*}$. In addition, the duration of the ELM magnetic fluctuations and their amplitude was smaller at low $\rho^{*}$. For the narrower pressure gradient region in the low $\rho^{*}$ case, the calculated edge bootstrap current profile in the equilibrium reconstruction was narrower than at higher $\rho^{*}$. Combining these in the peeling-ballooning stability calculation produced a higher toroidal mode number for the most unstable mode and, consequently, a prediction of a narrower ELM onset region at low $\rho^{*}$. For these similarity experiments, the discharges at reduced $\rho^{*}$ (by increased $B_{\mathrm{T}}$ ) also were at higher density, $n^{\text {ped }} \sim \boldsymbol{B}_{\mathrm{T}}{ }^{\prime-}$. Therefore, the narrowing of the steep gradient of the pressure was due in part to reduced neutral penetration at high density in this $\rho^{*}$ scan.

\subsection{ELM Dynamics in the Pedestal and Midplane SOL}

Midplane and SOL ELM dynamics measurements showed large, rapid variations of the SOL parameters and suggested a filamentary structure of the perturbation with fast radial propagation in later phases, and parallel propagation of the ELM pulse at speeds approaching the sound speed of pedestal ions. Previous measurements confirmed the expected outer midplane dominated peeling-ballooning spatial structure at ELM onset [19,20]. A reduction of $n_{\mathrm{e}}^{\mathrm{rcu}}$ was seen at all densities during an ELM and $T_{\mathrm{e}}^{\mathrm{rcu}}$ was also reduced at low $\mathrm{n}_{\mathrm{e}}^{\text {pcu }}$ ("conductive" ELMs) but no change to $T_{\mathrm{e}}^{\mathrm{pcu}}$ was seen during ELMs at high density ("convective" ELMs) [21]. Scanning reflectometer data showed that the particles lost from the pedestal during an ELM appeared far out in the SOL at the midplane [22]. This result was independent of the pre-ELM density. In the far outer SOL where $n_{\mathrm{e}}^{\text {DUL increases }}$ substantially, no increase in $T_{\mathrm{e}}^{\mathrm{JUL}}$ was observed, implying rapid parallel conduction of the ELM energy in the SOL. Fast CER measurements showed similar loss of impurities from the pedestal, a drop in pedestal toroidal and poloidal rotation, and the elimination of the pedestal electric field well by the ELM crash [23]. Scanning probe data near the separatrix showed large, rapid variations of both $\mathrm{n}_{\mathrm{e}}^{\mathrm{SOL}}$ and $T_{\mathrm{e}}^{\mathrm{SUL}}$ during ELMs suggesting a filamentary structure of the perturbation [ 24 ]. This interpretation was supported by recent data velocity of the density perturbation, inferred from an ultra-fast radial interferometer chord $\quad \mathrm{f} \quad \mathrm{r} \quad \mathrm{O} \quad \mathrm{m} \quad \mathrm{t} \quad \mathrm{h} \quad \mathrm{e}$ (Fig. 6) At the time of the ELM crash, the line integrated density at the midplane showed a burst of high frequency oscillations for $\approx 100 \mu \mathrm{s}$, consistent with the duration of the ELM perturbation on the fast magnetics signals. Beam emission spectroscopy (BES) data (Fig. 7) [24] also showed the development of a poloidally localized density "finger" that breaks away from the pedestal at the ELM crash. Finally, CIII $(465 \mathrm{~nm})$ visible emission data from a tangentially viewing fast-gated camera [25] at the midplane (Fig. 8), showed multiple filaments extended along the SOL flux surfaces that are consistent with a toroidal mode structure at $n \sim 18$ and $q \sim 4$. CER measurements indicated that the ELM density perturbation structure may be toroidally rotating in the SOL [26]. The radial velocity of the density perturbation, inferred from both the probe and reflectometer data, was $\sim 700 \mathrm{~m} / \mathrm{s}$ near the separatrix. The radial velocity decreased rapidly with radius in the SOL. Parallel 


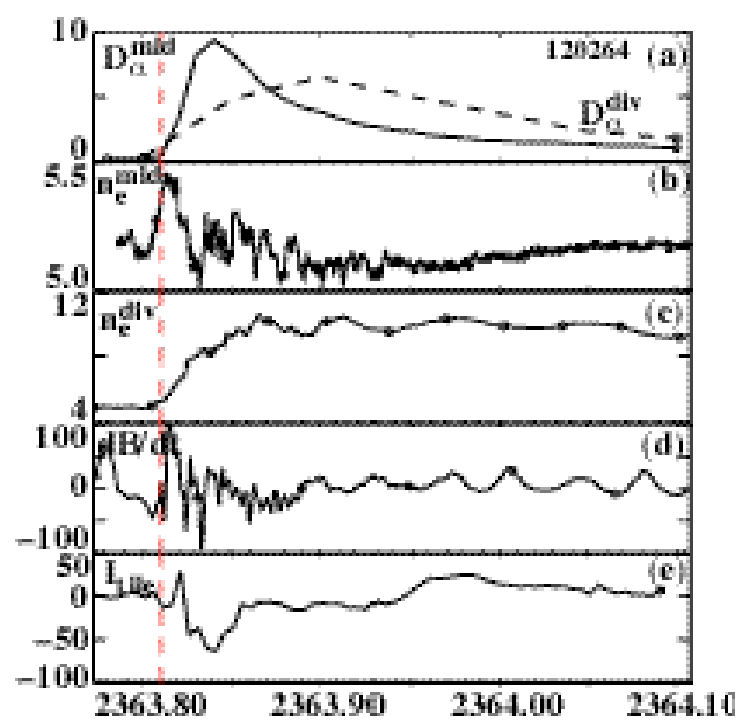

Fig. 6. Fast measurements of midplane dynamics during an ELM crash; (a) midplane and divertor $D_{\alpha}$ emission, (b) line averaged midplane density from radial interferometer chord $\left(10^{19} \mathrm{~m}^{-3}\right)$, (c) line averaged divertor density from a vertical interferometer chord $\left(10^{19} \mathrm{~m}^{-3}\right),(\mathrm{d}) \mathrm{dB} / \mathrm{dt}(\mathrm{T} / \mathrm{s})$, (e) current integrated on divertor tile (A). Two percent of the data points are marked with circles to indicate the temporal resolution. 
relative timing of the $D_{\alpha}$ pulses in the two divertors, approached the sound speed of ions at the pedestal temperature [27].

Poloidal and toroidal narrowing of the density perturbation into filaments (Figs. 9 and 10) were also seen in nonlinear ELM simulations [28] with the BOUT code [29]. These simulation used conditions of a high density DIII-D discharge with small, convective ELMs. ELITE indicated that the starting conditions were beyond the linear instability threshold. The projection of the density perturbation onto a poloidal plane [Fig. 9(a)] in the early phase of the nonlinear simulation shows the outer midplane dominated structure expected from peeling-ballooning theory. At this stage the perturbation has a toroidal mode number, $\mathrm{n} \sim 20$ [Fig. 10(a)] and has a linear growth rate normalized to the Alfven frequency of $\gamma / \omega_{\mathrm{A}} \sim 0.15$. When the growth becomes nonlinear, the density perturbation becomes very toroidally and poloidally localized, and finally bursts into the SOL and breaks into filaments [Figs. 9(b) and $10(b, c)]$, consistent with non-liner ballooning theory [30]. The simulation shows a substantial pedestal density drop and density increase in the far SOL at the crash, consistent with measurements.

\section{Summary and Conclusions}

Significant progress has been made toward a quantitative physics understanding that will increase confidence in our ability to predict two critical aspects of future high-power tokamak operation, namely the width of the density pedestal and the pedestal pressure gradient at

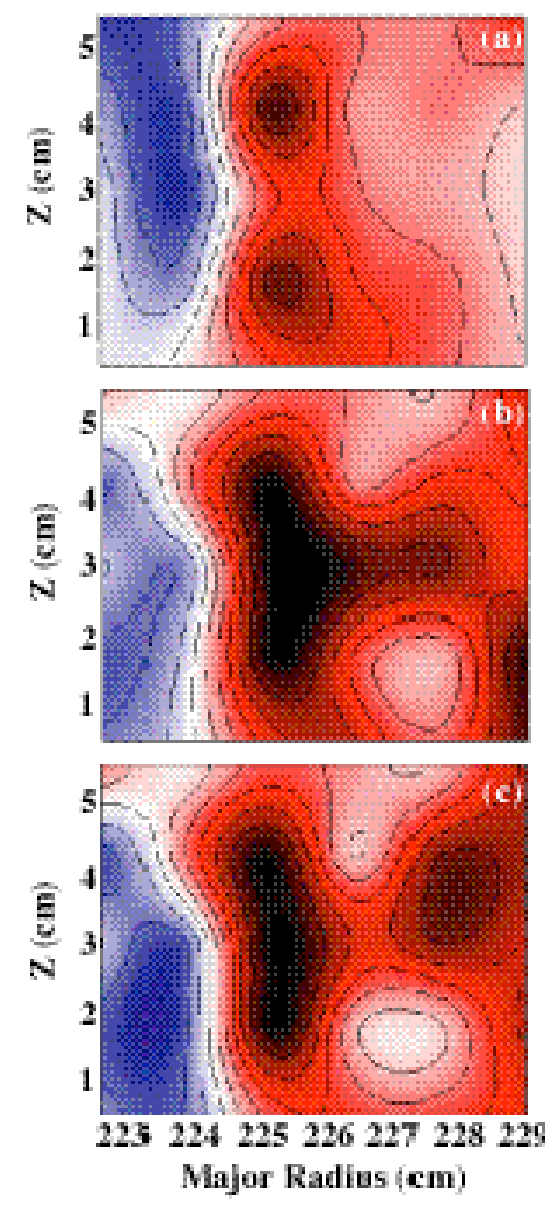

Fig. 7. Deviation of density (red-positive, blue-negative) from average (white) near the poloidal midplane (a) between ELMs, and $(b, c)$ during ELM build-up and crash. The ELM perturbation is highly localized poloidally and propagates radially into the SOL. 
Type-I ELM onset. Progress has also been made toward understanding the complex coupling of transport and stability mechanisms that set the temperature pedestal

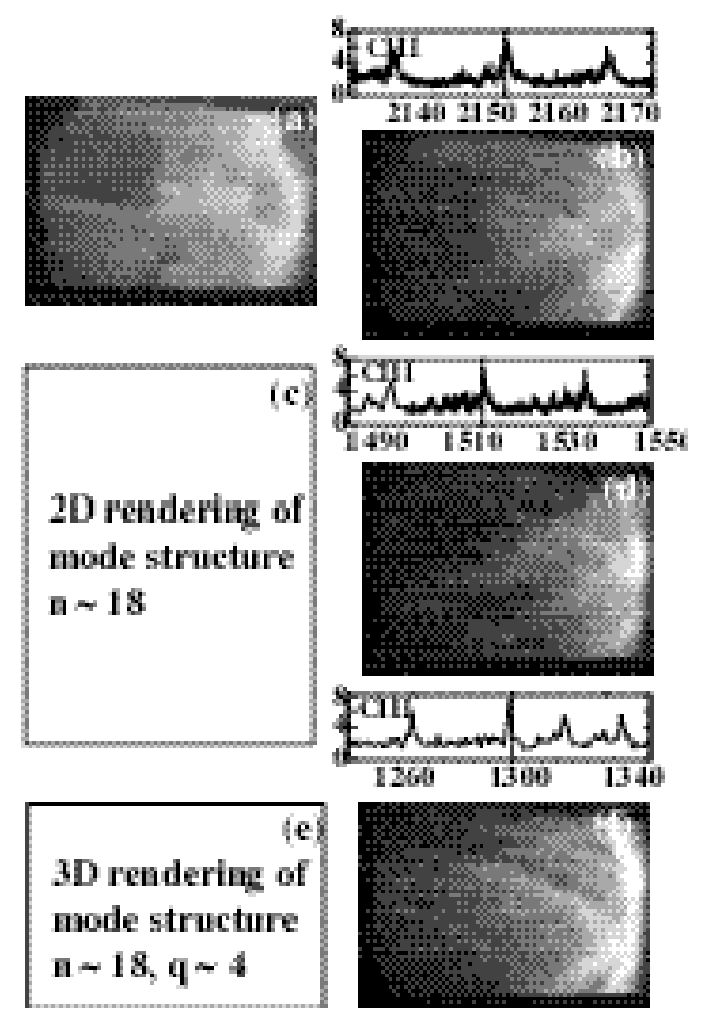

Fig. 8. CIII (465 nm) images with $10 \mu \mathrm{s}$ exposure during ELM crashes and ELITE simulation results. (a) Camera view of vacuum vessel in reflected light, $(b, d, f)$ images of CIII emission during different ELM crashes, (c) 2D profile of instability mode from ELITE for case shown in (f), (e) camera view of 3D mode structure from ELITE for case (f) 


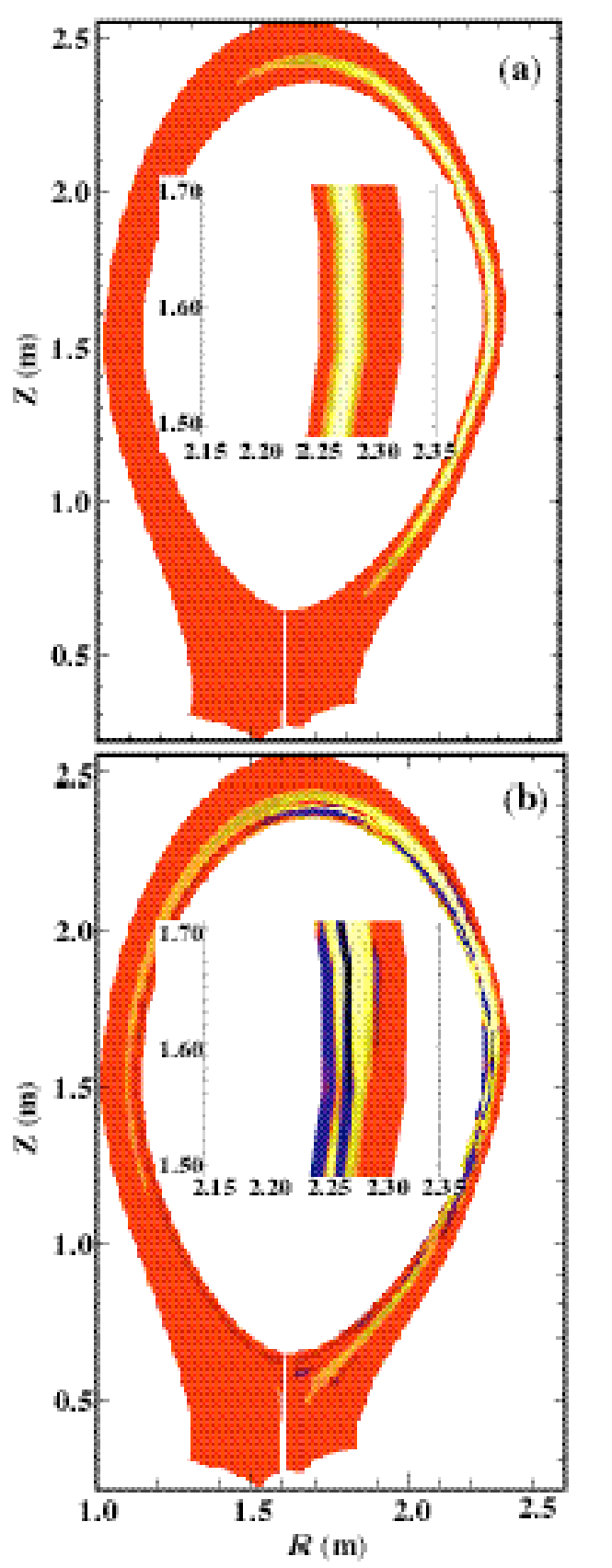

Fig. 9. Density perturbation projected onto a poloidal plane from (a) linear growth phase and (b) nonlinear crash phase of BOUT non-linear ELM simulation. Inset shows expansion of region near the outer midplane.
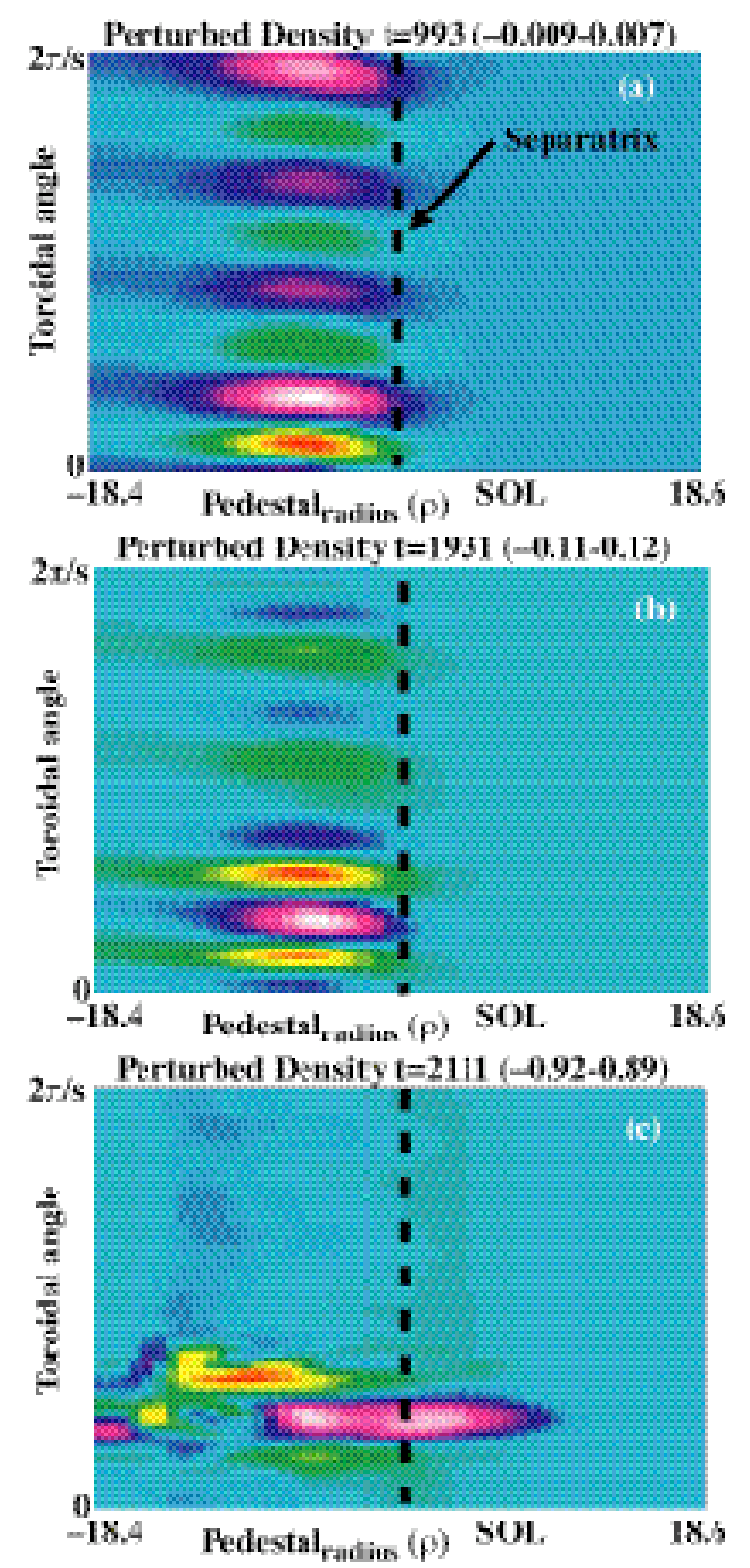

Fig. 10. Density contours vs. toroidal angle and radius from BOUT nonlinear ELM simulation showing (a) instability mode structure during linear growth phase, $(b, c)$ nonlinear growth of toroidally localized density perturbation and radial propagation at ELM crash.

height and width. Given knowledge of $n_{\mathrm{e}}^{\text {ped }}, \Delta_{\mathrm{n}}$ predicted from a neutral penetration model agrees with present measurements. The pedestal $\Delta_{\mathrm{T}}$ is set by plasma physics transport mechanisms not neutral penetration physics. The results suggest that it may be possible to independently control $\Delta_{\mathrm{n}}$, by controlling neutral sources. Independent control of the edge density profile at fixed temperature profile could allow optimization of the edge bootstrap current to minimize ELM energy loss for a given core confinement. Linear peelingballooning stability, calculations using a model of the edge bootstrap current constrained by $j$ edge measurements, predict instability of intermediate-n peeling-ballooning modes for the measured pedestal pressure at ELM onset. They also predict that lower edge current might increase the toroidal mode number of the most unstable mode leading to smaller ELMs. The reduction of measured ELM energy loss with decreasing $\rho^{*}$ in the similarity experiments was 
consistent with increased n-number of the most unstable mode leading to narrower ELM affected region in the edge. This suggests that tolerable sized ELMs may be possible in future devices at low $\rho^{*}$ and high density. In addition, the lack of $\rho^{*}$ dependence of $\Delta_{\mathrm{T}} / a$ also suggests favorable confinement in future devices with small $\rho^{*}$. Finally, recent fast measurements of ELM dynamics in the midplane pedestal and SOL show evidence for a filamentary structure of the perturbation at the nonlinear ELM crash. Initial non-linear fluid simulations show a poloidally and toroidally localized density perturbation at the crash leading to a filamentary structure in the SOL, in qualitative agreement with the data.

\section{Acknowledgments}

This work was performed under the auspices of the U.S. Department of Energy by University of California Lawrence Livermore National Laboratory under Contract No. W7405-ENG-48, DE-FC02-04ER54698, DE-AC05-00OR22725, and Grants DE-FG0204ER54758, DE-FG03-95ER54309, and DE-FG03-01ER54615.

\section{References}

[1] OSBORNE, T.H., T.H., et al., Plasma Phys. and Control. Fusion 42 (2000) 1.

[2] FEDERICI, G., et al., J. Nucl. Mater. 313-316 (2003) 11.

[3] THOMAS, D.M., et al., Phys. Rev. Lett. 96 (2004) 65003.

[4] COLCHIN, R.J., et.al., Rev. Sci. Instrum. 74 (2003) 2068.

[5] BOEDO, J.A., et al., Rev. Sci. Instrum. 69 (1998) 2663; BOEDO, J.A., et al., Rev. Sci. Instrum. 70 (1999) 2997.

[6] ZENG, L., et al., Rev. Sci. Instrum. 74 (2003) 1530; WANG, G., et al., Rev. Sci. Instrum. 74 (2003) 1525.

[7] McKEE, G.R., et al., Rev. Sci. Instrum. 70 (1999) 2179; McKEE, G.R., et al., Rev. Sci. Instrum. 74 (2003) 2014

[8] VANZEELAND, M.A., and CARLSTROM, T.N., accepted for publication in Rev. Sci. Instrum. 75 (2004); VANZEELAND, M.A., private communication.

[9] WAGNER, F., and LACKNER, K., Phys. Plasmas-Wall Interactions in Controlled Fusion, NATO ASI Series B, Physics, Vol. 131, p. 931.

[10] MAHDAVI, M.A., et al., Phys. Plasmas 10 (2003) 3988.

[11] HINTON, F.L., and STAEBLER, G.M., Phys. Fluids B5 (1993) 1281.

[12] MOSSESSIAN, D.A., SNYDER, P.B., HUBBARD, A., et al., Phys. Plasmas 10 (2003) 1720; MOSSESSIAN, D.A., Proc of the 30th EPS Conf. on Control Fusion and Plasma Phys., St. Petersburg, Russia ECA Vol. 27A (2003) P3.182

[13] THOMAS, D.M., et al., Proc of the 31st EPS Conf. on Control Fusion and Plasma Phys., London, England ECA Vol. 28G, (2004) P2.177

[14] THOMAS, D.M., et al., Rev Sci. Instrum. 74 (2003) 1541; THOMAS, D.M., et al., Rev. Sci. Instrum., in press (2004).

[15] HOUlBERG, W.A., et al., Phys. Plasmas 4 (1997) 3230.

[16] CASPER, T.A., et al., Proc of the 30th EPS Conf. on Control Fusion and Plasma Phys., St. Petersburg, Russia (2003) P3.207.

[17] SNYDER, P.B., et al., Phys. Plasmas 9 (2002) 2037; SNYDER, P.B., et al., Nucl. Fusion 44 (2004) 320.

[18] WILSON, H.R., et al., Phys. Plasmas 9 (2002) 1277.

[19] MAHDAVI, M.A., et al., J. Nucl. Mater. 162-164 (1988) 245.

]20] PETRIE, T.W., et al., Nucl. Fusion 43 (2003) 910.

[21] LEONARD, A.W., et al., J. Nucl. Mater. 313-316 (2003) 768; LEONARD, A.W., et al., Phys Plasmas 10 (2003) 1765.

[22] ZENG, L., et al., Plasma Phys. Control. Fusion 46 (2004) A121; ZENG, L., et al., "SOL and pedestal density evolution in DIII-D H-mode.” accepted for publication in J. Nucl. Mater. (2004).

[23] WADE, M.R., et al., "Time resolved measurement of the impurity, ion, and electron dynamics using an ELM cycle,” Phys. Rev. Lett., submitted (2004).

[24] BOEDO, J.A., et al., "ELM dynamics and transport in the SOL of the DIII-D tokamak," submitted for publication in Phys. Rev. Lett. (2004).

[25] GROTH, M., et al., Rev. Sci. Instrum. 74 (2003) 2064.

[26] BOEDO, J.A., et al., "ELM-induced plasma transport in the DIII-D SOL," Proc. of the 31st EPS Conf. on Control Fusion and Plasma Physics, London UK (2004).

[27] FENSTERMACHER, M.E., et al., Plasma Phys. Control. Fusion 45 (2003) 1597; FENSTERMACHER, M.E., et al., accepted for publication in J. Nucl. Mater. (2005). 
[28] SNYDER, P.B., WILSON, H.R., Xu. X.Q., and WEBSTER, A.J., "Progress in the peeling-ballooning model of ELMs: toroidal rotation and 3D nonlinear dynamics," Proc of the 31st EPS Conf. on Control Fusion and Plasma Phys., London, England (2004).

[29] XU, X.Q., et al., Nucl. Fusion 40 (2000) 731.

[30] WILSON, H.R., and COWLEY, S.C., Phys. Rev. Lett. 92 (2004) 175006. 